\title{
Chapter 20 \\ Evaluation of the Length of Hospital Stay Through Artificial Neural Networks Based Systems
}

\author{
Vasco Abelha \\ University of Minho, Portugal \\ Fernando Marins \\ University of Minho, Portugal \\ Henrique Vicente \\ University of Evora, Portugal
}

\begin{abstract}
The mentality of savings and eliminating any kind of outgoing costs is undermining our society and our way of living. Cutting funds from Education to Health is at best delaying the inevitable "Crash" that is foreshadowed. Regarding Health, a major concern, can be described as jeopardize the health of Patients - Reduce of the Length of Hospital. As we all know, Human Health is very sensitive and prune to drastic changes in short spaces of time. Factors like age, sex, their ambient context-house conditions, daily lives - should all be important when deciding how long a specific patient should remain safe in a hospital. In no way, ought this be decided by the economic politics. Logic Programming was used for knowledge representation and reasoning, letting the modeling of the universe of discourse in terms of defective data, information and knowledge. Artificial Neural Networks and Genetic Algorithms were used in order to evaluate and predict how long should a patient remain in the hospital in order to minimize the collateral damage of our government approaches, not forgetting the use of Degree of Confidence to demonstrate how feasible the assessment is.
\end{abstract}

